\title{
Acceptability of diagnostic tests for breast cancer
}

\author{
Wenchi Liang ${ }^{1}$, William F. Lawrence ${ }^{2}$, Caroline B. Burnett ${ }^{1,3}$, Yi-Ting Hwang ${ }^{1,6}$, Matthew \\ Freedman ${ }^{4}$, Bruce J. Trock ${ }^{5,7}$, Jeanne S. Mandelblatt ${ }^{2}$, and Marc E. Lippman ${ }^{2,8}$ \\ ${ }^{1}$ Department of Oncology; ${ }^{2}$ Departments of Medicine and Oncology; ${ }^{3}$ School of Nursing; ${ }^{4}$ Imaging Science and \\ Information Systems Center; ${ }^{5}$ Department of Biomathematics and Biostatistics, Georgetown University Medical \\ Center, Georgetown University, Washington, USA; Present addresses: ${ }^{6}$ Department of Statistics, National Taipei \\ University; ${ }^{7}$ Department of Urology, Johns Hopkins Medical Institutions; ${ }^{8}$ Department of Internal Medicine, \\ University of Michigan, USA
}

Key words: biopsy, breast cancer, breast magnetic resonance imaging, digital mammography, satisfaction, Tc-99msestamibi scanning, test acceptability, willingness to pay

\section{Summary}

Purpose. To assess the acceptability of new non-invasive breast cancer diagnostic tests intended to triage women in need of biopsy.

Methods. Women who had abnormal screening tests and had been recommended to have a biopsy were invited to receive digital mammography, magnetic resonance imaging (MRI), and nuclear medicine evaluation (Tc-99msestamibi scanning) before biopsy. Participants completed a questionnaire about satisfaction and acceptability of the procedures. Satisfaction measured women's overall and test-specific satisfaction. Acceptability was measured by self-reported discomfort, embarrassment and women's preference in terms of willingness to pay to avoid a biopsy.

Results. Women were satisfied with all of the potential diagnostic triage procedures. Most found the tests more comfortable than a routine mammogram $(47,50$, and $66 \%$ undergoing MRI, digital mammography, and sestamibi scanning, respectively). Women who provided a response to willingness to pay questions $(N=43)$ were willing to pay an average of $\$ 611$ to have a test instead of a biopsy, if the test was as accurate as biopsy. The willingness to pay significantly decreased to $\$ 308$ if the test only had $95 \%$ accuracy. Those who had prior benign breast disease were less willing to pay for a test with $95 \%$ accuracy than those without this history.

Conclusion. Instead of immediate biopsy after an abnormal screening, these results suggest that women would find non-invasive triage tests acceptable, or preferable to biopsy if they were equally accurate or nearly equally accurate as a biopsy. New technologies to diagnose breast cancer should focus on decreasing discomfort as well as increasing test accuracy.

\section{Introduction}

There are more than 600,000 breast biopsies performed annually in the US [1]; as many as $80 \%$ of these yield benign results [2,3]. While biopsies are the definitive tests for diagnosing cancer, they are invasive, and can be uncomfortable and anxiety provoking. Thus, alternative, non-invasive diagnostic pathways could have large potential economic and quality of life impact $[4,5]$.
However, when making decision on using noninvasive diagnostic tests, women must balance the concerns of discomfort and anxiety from biopsies against the possibility of reduced accuracy of the noninvasive tests. A false positive result would result in a biopsy anyway, so the diagnostic pathway would include one more test than if the woman had a biopsy immediately following the abnormal mammogram or breast examination. Perhaps of more concern to women would be a false negative on the new test, missing a 
breast cancer resulting in a delay in diagnosis. Thus, as novel diagnostic tests are introduced into practice, women must balance the benefits of potentially avoiding a biopsy with the risks of a potentially less accurate diagnostic test.

Although new non-invasive technologies are being developed to increase the accuracy of detecting breast cancer [6-9], there are no studies that we are aware of that have examined the acceptability of these technologies from the perspective of patients who receive them. The purpose of this project is two-fold. First, among a cohort of women who had an abnormal breast finding and were recommended to have a biopsy, we assess the patient's perspective on the acceptability of new diagnostic radiology technologies. Our second objective is to understand how test accuracy is related to women's preferences for avoiding a biopsy in favor of a non-invasive diagnostic test.

\section{Methods}

\section{Study sample and procedures}

We prospectively enrolled a cohort of white and African-American women from several DCmetropolitan area clinics, hospitals, and HMOs, who had abnormal breast physical examination, mammography, and/or clinical ultrasound examination (usually done on the identified palpable abnormality and performed without Doppler imaging) results and had been recommended to have a breast biopsy between June 1998 and October 2000. After providing informed consent, participants received a series of breast cancer diagnostic tests, including digital mammography, magnetic resonance imaging (Gd-DTPA enhanced MRI), and nuclear medicine evaluation (Tc-99m-sestamibi scanning). A short selfadministered questionnaire about satisfaction and acceptability of the tests was given to women after completion of all tests. Both the diagnostic tests and the survey were done on the same day visit, which was scheduled prior to receiving their breast biopsy. To minimize test selection bias, women were asked to receive all the diagnostic tests and no information on the sensitivity and specificity of individual tests were given at the consenting process. Failure to receive certain tests was largely due to unavailability of those tests on the day of visit (e.g., equipment breakage and scheduling conflicts).

\section{Measures}

Patient characteristics included age, monthly household income, previous breast biopsy, history of benign breast disease, history of breast cancer, family history of breast cancer, and women's perceived risk of getting breast cancer in the future.

Satisfaction with participating in this study and with the overall experience of receiving each individual diagnostic test was measured using a modification of the Medical Outcomes Study Visit Rating Questionnaire [10]. The six satisfaction questions measured satisfaction with the receipt of the tests overall, the technical skill of the staff, the personal manner of the staff, the convenience of getting the tests, the length of time spent waiting for the test, and the explanation of what was done for the participants. Responses to each question were rated on a five-item Likert scale.

Discomfort associated with having a routine mammogram was measured using a five-item Likert scale (extremely, very, somewhat, mildly, and not uncomfortable at all). To provide a relative standard, we asked the participants to rate discomfort of other tests compared to having a routine mammogram (a lot less, a little less, no different, a little more, a lot more). Embarrassment was measured using a fouritem Likert scale (extremely, somewhat, mildly, and not embarrassing at all) for each test.

The willingness to pay technique was used to assess women's preferences for having one of the non-invasive diagnostic tests compared to a surgical biopsy. We used the willingness to pay technique as a process utility, or a quantitative measure of preference that may be applied to the process of care [11-15]. The willingness to pay questions ask respondents how much money they think women like themselves would be willing to pay out-of-pocket for an alternative non-invasive diagnostic test instead of a biopsy. We worded these questions in the third person to reduce the potential for anxiety about breast cancer in women undergoing evaluation for a suspicious breast lesion. Two willingness to pay scenarios were used to evaluate the impact of diagnostic accuracy on preferences. The first asks about willingness to pay to have a test instead of a biopsy, if the test were as accurate as a biopsy at diagnosing cancer. The second asks about willingness to pay if the test were nearly $(95 \%)$ as accurate as a biopsy. The reason for using $95 \%$ accuracy rate was to assess how preference changes for tests that are slightly less accurate than the 
'gold standard', in this case, breast biopsy. We asked participants to imagine whichever new test they would most prefer having, to avoid the respondent burden of asking about each test separately. Thus, the assessment provides the maximum the respondent would be willing to pay for any of the tests. Since the willingness to pay technique is sensitive to economic status, willingness to pay was further defined as the amount a woman thinks women like her would be willing to pay as a proportion household income.

\section{Data analysis}

Analyses were performed using the SAS System version 8. Patient satisfaction, discomfort, and embarrassment scales were analyzed by frequencies and the non-parametric Kruskal-Wallis tests for difference in distribution. The differences in willingness to pay by patient characteristics were presented by the average amount of money women would be willing to pay in individual scenarios and the ratio of the result for the two scenarios using $t$ tests and ANOVA tests. Because of the small sample size and non-normal distribution of the ratio between willingness to pay and income, the non-parametric Kruskal-Wallis test was used to test the difference in willingness to pay by patient characteristics. Finally, the difference in the ratio between the two willingness to pay values (95 v.s. $100 \%$ accuracy) by patient characteristics was analyzed using the non-parametric sign test.

\section{Results}

\section{Participant characteristics}

A total of 106 patients were recruited for this study, $82(77 \%)$ of which completed the survey. Those who completed the survey were similar with respect to demographics and cancer history as those who did not complete the survey. The mean age of this sample was $51.6($ range $=25-78)($ Table 1$)$. Eighty percent were white, and 14\% were African Americans. About 23\% had a monthly household income of up to $\$ 3000$, and 13 and 64\% had an income level of \$3000-4999 and $\$ 5000$ and above, respectively. Prior to entry into this study, about $46 \%(N=37)$ had a history of a prior breast biopsy; 10 of these had benign breast disease, two were diagnosed with ductal carcinoma in situ (DCIS), one had lobular carcinoma in situ (LCIS), nine had other types of breast malignancy, and 15 did not report having any kind of breast disease. Of
Table 1. Participant characteristics $(N=82)$

\begin{tabular}{ll}
\hline Patient characteristics & Distribution \\
\hline Age & \\
$\quad 40$ and younger & $13.6 \%$ \\
$41-50$ & $32.1 \%$ \\
$51-60$ & $37.0 \%$ \\
61 and older & $17.3 \%$ \\
Mean age (range) & $51.6(25-78)$ \\
Race & \\
White & $80.2 \%$ \\
African American & $14.5 \%$ \\
Hispanic & $1.3 \%$ \\
Asian & $2.6 \%$ \\
Other & $1.3 \%$ \\
Household income & \\
Less than $\$ 1000$ & $3.1 \%$ \\
\$1000 to $\$ 1999$ & $6.3 \%$ \\
\$2000 to $\$ 2999$ & $14.1 \%$ \\
\$3000 to $\$ 3999$ & $1.6 \%$ \\
\$4000 to \$4999 & $10.9 \%$ \\
\$5000 or more & $64.1 \%$ \\
Previous breast biopsy & $46.3 \%$ \\
History of benign breast disease & $21.3 \%$ \\
History of DCIS & $2.6 \%$ \\
History of LCIS & $1.3 \%$ \\
History of breast malignancy & \\
other than LCIS and DCIS & $11.4 \%$ \\
Family history of breast cancer & $45 \%$ \\
\hline
\end{tabular}

the $17(21.3 \%)$ women with a benign breast disease, seven did not receive a breast biopsy prior to the study. Thirty-six participants had a family history of breast cancer, and two had a family history of ovarian cancer.

\section{Satisfaction with care}

In general, participants were very satisfied with the process of receiving the different diagnostic tests (Table 2). About $96 \%$ of patients gave an excellent or very good rating of the overall process of participating in the study.

\section{Acceptability - degree of discomfort}

About $20 \%$ of the participants reported that a routine mammogram made them very or extremely uncomfortable, and another $20 \%$ reported mammography as not uncomfortable at all (Figure 1). Among those receiving the tests, $47 \%$ of those receiving MRI, $50 \%$ 
Table 2. Patient satisfaction with the diagnostic process

\begin{tabular}{lcccccc}
\hline & Overall & Technical skills & Personal manner & Convenience & Time spent in waiting & Explanation \\
\hline$N$ & 79 & 66 & 80 & 81 & 79 & 81 \\
Excellent (\%) & 73 & 83 & 89 & 43 & 62 & 80 \\
Very good (\%) & 23 & 15 & 9 & 40 & 28 & 16 \\
Good (\%) & 1 & 1 & 1 & 14 & 5 & 4 \\
Fair (\%) & 3 & 1 & 1 & 1 & 5 & 0 \\
Poor (\%) & 0 & 0 & 0 & 0 & 0 \\
\hline
\end{tabular}

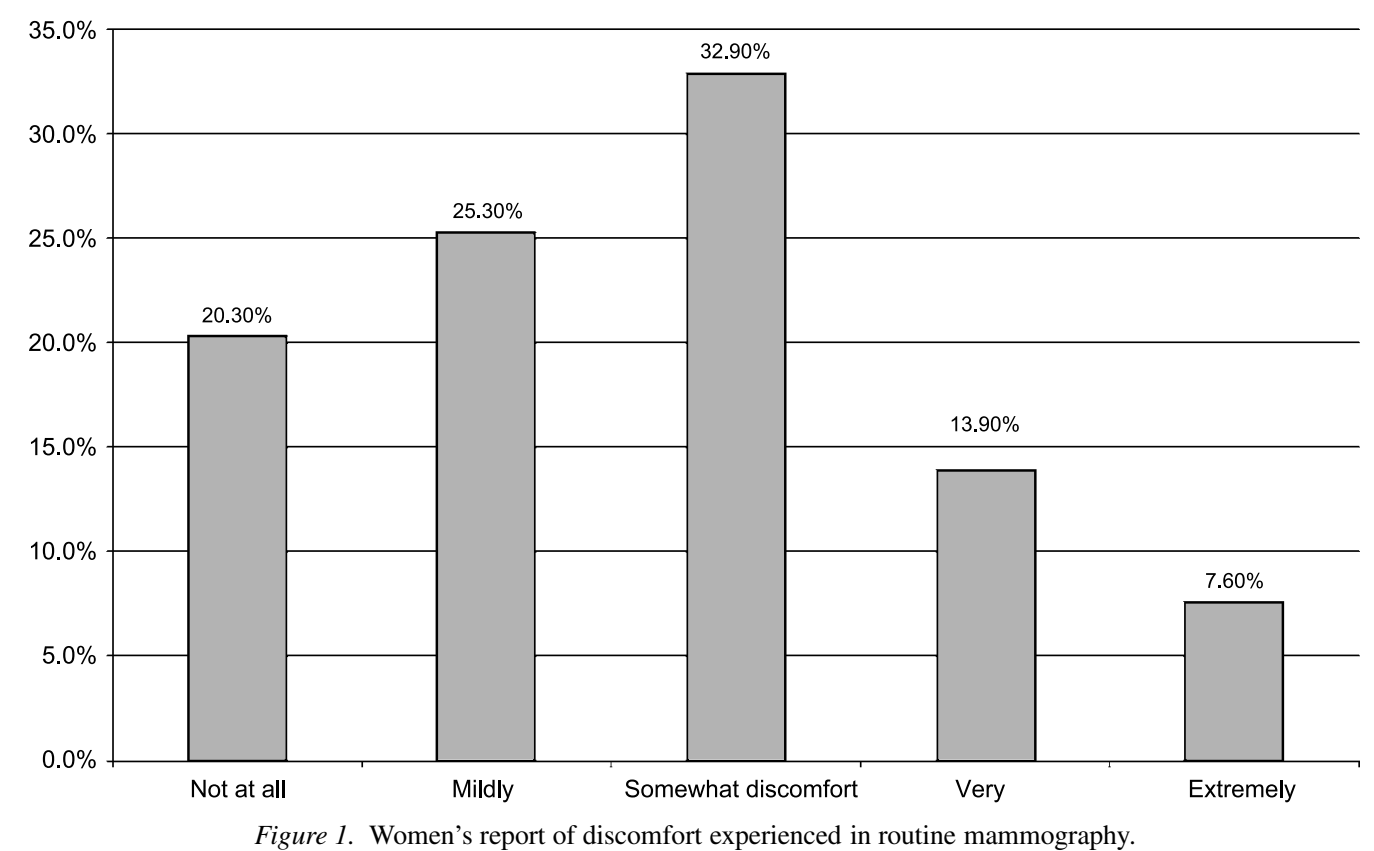

of those receiving digital mammography, and $66 \%$ of those receiving sestamibi imaging found the procedure more comfortable than a routine mammogram (Table 3). Compared to routine mammography, the sestamibi nuclear medicine test was perceived as the most comfortable test.

\section{Acceptability-degree of embarrassment}

Most of the participants did not feel embarrassed when receiving any of the diagnostic tests (Table 4).

\section{Preference - willingness to pay}

Under conditions of equal accuracy to a biopsy, women were willing to pay an average of $\$ 611$ to have a non-invasive test instead of a biopsy (range $\$ 0-10,000$ ), with $7 \%$ of women not willing to pay any money out of pocket (Table 5). The willingness to pay significantly decreased to an average of $\$ 308$ in the case of $95 \%$ accuracy (range \$0-3000), with 33\% of women not willing to pay any money $(p<0.0001)$. This decrease of willingness to pay was consistent across patient characteristics.

Regarding the difference in willingness to pay by patient characteristics, women who had a history of benign breast disease tended to be willing to pay less to avoid a biopsy than women without history of breast disease. There was no difference in willingness to pay by age, perceived vulnerability of breast cancer, household income, history of biopsy, history of breast cancer, and family history of breast cancer (Table 5). However, interesting non-significant trends were observed in several categories: Women ages 60 and older were less willing to pay out of their own pockets for diagnostic tests (e.g., willing to pay \$220 compared 
Table 3. Discomfort experienced from diagnostic tests compared to a routine mammogram

\begin{tabular}{llll}
\hline $\begin{array}{l}\text { Discomfort compared to routine } \\
\text { mammogram (score) }\end{array}$ & MRI [55 (67\%)] & $\begin{array}{l}\text { Digital mammogram } \\
{[14(17 \%)]}\end{array}$ & $\begin{array}{l}\text { Nuclear medicine } \\
{[71(87 \%)]}\end{array}$ \\
\hline A lot less (2) & $27 \%$ & $14 \%$ & $45 \%$ \\
A little less (1) & $20 \%$ & $36 \%$ & $21 \%$ \\
No different (0) & $15 \%$ & $50 \%$ & $13 \%$ \\
A little more (-1) & $16 \%$ & - & $18 \%$ \\
A lot more (-2) & $22 \%$ & - & $3 \%$ \\
Mean score & 0.15 & 0.64 & 0.87 \\
\hline
\end{tabular}

Table 4. Embarrassment experienced from diagnostic tests

\begin{tabular}{llccc}
\hline Embarrassment level & Routine mammogram & MRI & Digital mammogram & Nuclear medicine \\
\hline$N$ & 80 & 53 & 14 & 72 \\
Not embarrassing at all (\%) & 76 & 87 & 93 & 83 \\
Mildly embarrassing (\%) & 11 & 11 & 7 & 11 \\
Somewhat embarrassing (\%) & 11 & 0 & 0 & 4 \\
Extremely embarrassing (\%) & 1 & 2 & 0 & 1
\end{tabular}

Note: Kruskal-Wallis tests showed no significant difference among routine mammogram, MRI, digital mammogram, and nuclear medicine.

to \$962 among women aged 50-59). Those who had previous biopsy with benign results were willing to spend more for other non-invasive tests (willing to pay $\$ 589$ ); whereas those with a history of abnormal biopsy results would pay less for diagnostic tests other than biopsy (willing to pay $\$ 116$ ). Women with breast cancer or family history of breast cancer were less willing to pay for other diagnostic tests (willing to pay $\$ 200$ and $\$ 236$, compared to $\$ 315$ and $\$ 377$ among those without history or family history of breast cancer).

\section{Discussion}

This study demonstrated that new non-invasive breast cancer diagnostic tests (digital mammography, MRI, and sestamibi nuclear medicine) are generally acceptable (in terms of level of discomfort and embarrassment) to women who have abnormal breast findings and for whom a breast biopsy is recommended. Women generally consider these new diagnostic tests more comfortable than a routine mammogram. The degree of embarrassment from taking these tests are similar to that seen with routine mammograms. Prior research shows that discomfort associated with mammography is a barrier to regular breast cancer screening [16, 17]. Decreasing women's physical discomfort while receiving breast cancer diagnostic tests may likewise be an important objective to ensure compliance with timely follow-up.

The acceptability of new diagnostic test is influenced by test accuracy. In our study, the importance of test accuracy was assessed by women's willingness to pay for the test out of their own pocket. A decrease of 5\% accuracy, from 100 to $95 \%$, of the preferred test resulted in a $40 \%$ decrease of willingness to pay, and the sizable decrease was consistent across different age, biopsy history, breast disease, family history of breast cancer, and risk perception groups. These results suggest that the accuracy of diagnostic tests may be more important than the level of discomfort and embarrassment associated with a test. Because test accuracy will have impact on long-term health outcomes, women may prefer a more uncomfortable but more accurate test to avoid getting false diagnosis.

Moreover, the willingness to pay measure showed that women with previous benign breast disease tended to be more willing to pay for new diagnostic tests to avoid unnecessary biopsies than those with no benign breast disease. It is possible that a benign disease history is acting as a proxy for experience with breast biopsies, alternatively, women with prior benign 
Table 5. Willingness to pay according to accuracy of diagnostic tests, by patient characteristics

\begin{tabular}{|c|c|c|c|}
\hline & $100 \%$ accuracy (A) Mean (SD) & 95\% accuracy (B) Mean (SD) & Ratio (B/A) Mean (SD) \\
\hline Overall mean $(\mathrm{SD})^{\mathrm{a}}$ & $\$ 611(1462)$ & $\$ 308(561)$ & $0.60(0.48)$ \\
\hline Range & $\$ 0-10,000$ & $\$ 0-3000$ & $0-1$ \\
\hline \multicolumn{4}{|l|}{ Chance of getting breast cancer } \\
\hline Much higher & $\$ 439(453)$ & $\$ 268(340)$ & $0.60(0.45)$ \\
\hline A little higher & $\$ 428(475)$ & $\$ 300(509)$ & $0.58(0.45)$ \\
\hline The same or less & $\$ 1245(3090)$ & $\$ 465$ (909) & $0.67(0.37)$ \\
\hline ANOVA test $p$ value & 0.37 & 0.70 & 0.87 \\
\hline \multicolumn{4}{|l|}{ Household monthly income } \\
\hline$<\$ 3000$ & $\$ 446(519)$ & $\$ 169(223)$ & $0.58(0.49)$ \\
\hline$\$ 3000-4999$ & $\$ 775$ (1037) & $\$ 495(636)$ & $0.57(0.37)$ \\
\hline$>\$ 5000$ & $\$ 698(1774)$ & $\$ 368(651)$ & $0.60(0.43)$ \\
\hline ANOVA test $p$ value & 0.92 & 0.59 & 0.99 \\
\hline \multicolumn{4}{|l|}{ Age } \\
\hline Up to 40 & $\$ 371(372)$ & $\$ 292(304)$ & $0.72(0.40)$ \\
\hline $41-49$ & $\$ 411(629)$ & $\$ 304(403)$ & $0.71(0.40)$ \\
\hline $50-59$ & $\$ 962(2142)$ & $\$ 412(768)$ & $0.48(0.43)$ \\
\hline 60 and older & $\$ 220(193)$ & $\$ 58(68)$ & $0.62(0.49)$ \\
\hline ANOVA test $p$ value & 0.54 & 0.52 & 0.43 \\
\hline \multicolumn{4}{|l|}{ Previous biopsy } \\
\hline Yes & $\$ 777(2086)$ & $\$ 329(682)$ & $0.47(0.43)$ \\
\hline With normal results & $\$ 443(440)$ & $\$ 261(402)$ & $0.60(0.42)$ \\
\hline With benign results & $\$ 1714(3660)$ & $\$ 589$ (1096) & $0.33(0.47)$ \\
\hline With positive cell change ${ }^{b}$ & $\$ 183(194)$ & $\$ 116(194)$ & $0.46(0.42)$ \\
\hline No & $\$ 476(623)$ & $\$ 293(463)$ & $0.69(0.40)$ \\
\hline$t$-test $p$ value & 0.52 & 0.83 & 0.09 \\
\hline \multicolumn{4}{|l|}{ Benign breast disease $\mathrm{e}^{\mathrm{c}}$} \\
\hline Yes & $\$ 1325(2808)$ & $\$ 523(893)$ & $0.45(0.45)$ \\
\hline No & $\$ 379(463)$ & $\$ 238(395)$ & $0.65(0.41)$ \\
\hline$t$-test $p$ value & 0.27 & 0.31 & 0.17 \\
\hline \multicolumn{4}{|l|}{ Breast cancer } \\
\hline Yes & $\$ 267$ (208) & $\$ 200(265)$ & $0.50(0.50)$ \\
\hline No & $\$ 633(1056)$ & $\$ 315(576)$ & $0.60(0.43)$ \\
\hline$t$-test $p$ value & 0.16 & 0.73 & 0.69 \\
\hline \multicolumn{4}{|l|}{ Family history of breast cancer } \\
\hline Yes & $\$ 436(570)$ & $\$ 236(381)$ & $0.53(0.47)$ \\
\hline No & $\$ 778(1975)$ & $\$ 377(693)$ & $0.65(0.39)$ \\
\hline$t$-test $p$ value & 0.41 & 0.39 & 0.38 \\
\hline
\end{tabular}

SD: Standard deviation.

a The difference in the willingness to pay to income ratio between 100 and $95 \%$ accuracy groups was significant (sign test, $p<$ $0.0001)$.

$\mathrm{b}$ Including atypical hyperplasia, DCIS, LCIS, and malignancy.

${ }^{\mathrm{c}}$ The willingness to pay ratio (B/A) was significantly different between those having and not having benign breast disease in the non-parametric test, controlling for income (Kruskal-Wallis test, $p=0.027$ ).

disease may be more worried about cancer. These women may, judging from their experience, be more willing to accept new technologies if these tests can offer a confirmatory diagnosis and avoid unnecessary surgical procedures. This explanation can also be applied to our finding that those who had prior benign biopsy results were more willing to pay for new tests than those who had prior abnormal biopsy results. 
Women with personal or family history of breast cancer are more likely to favor biopsy and less likely to pay for new tests. This assumption is mirrored by our finding that women with high levels of risk perception were not as willing as those with lower levels of risk perception to pay for new tests, even if the tests are as good as biopsy in diagnosing breast cancer. Future research should examine the associations between disease history and risk perception and willingness to pay using a larger and diverse sample.

The interpretation of the results of this study is limited by several factors. First, the generalizability of our results is limited because we used a convenient sample and no information on the number of decliners and the reasons for refusals were available from the referring clinics. However, since the diagnostic tests were free of charge and scheduled on the same day, refusal rates were expected to be minimal. Second, the small sample size limited power to detect significant relationships and the ability to determine the relative importance of patient characteristics on willingness to pay. Third, women's understanding of each test may not have been sufficiently accurate, since about $50 \%$ considered digital mammography less uncomfortable than routine mammography when in fact the only difference is in imaging, and not in patient-related procedures. However, women might actually experience less discomfort in digital mammography than in previous mammography because they were more nervous or had less skilled technicians during previous mammography. On the other hand, a higher acceptability of digital mammography may simply reflect women's bias toward new technology. A more detailed questionnaire is needed to understand the reasons for rating discomfort and embarrassment. Fourth, the ratio of willingness to pay and income were crude because income was measured by category, not actual numerical values. Finally, the high levels of satisfaction we observed may have been affected by social desirability biases and may over estimate true satisfaction.

Despite these limitations, this study one of the first studies using a prospective cohort to assess the satisfaction and acceptability of new breast cancer diagnostic tests. We conclude that women would find these tests acceptable, and would find the tests preferable to biopsy if they were equally accurate or nearly equally accurate at evaluating breast lesions as biopsy. The development of novel breast cancer screening and diagnostic tests should focus on women's perceptions of comfort and preferences for outcomes.

\section{Acknowledgements}

This research was supported by U.S. Department of the Army Contract \#17-96-C-6069 and U.S. Department of the Army Grant \#17-94-J-4212. We appreciate the work of Anita Sarcone in patient recruitment, interviews, and arrangement of diagnostic tests. We also thank Michelle Brotzman for data management.

\section{References}

1. Osteen RT, Cady B, Chmiel JS: 1991 National survey of carcinoma of the breast by the commission on cancer. J Am Coll Surgeons 178: 213-219, 1991

2. Winchester DP, Senen S, Immerman S, Blum MA: A systematic approach to the evaluation and management of breast masses. Cancer 51: 2535-2540, 1983

3. Artz D, Blume E: Too many breast biopsies? Watching vs. cutting. J Natl Cancer I 83: 1207-1209, 1991

4. Gram IT, Lund E, Slenker SE: Quality of life following a positive mammogram. Brit J Cancer 62: 1108-1122, 1990

5. Parker RG: The 'cost-effectiveness' of radiology and radiologists. Radiology 189: 363-369, 1993

6. Klaus AJ, Kingensmith III WC, Parker SH, Stavros AT, Sutherland JD, Aldrete KD: Comparative value of $99 \mathrm{mTc}-$ sestamibi scintimammography and sonography in the diagnostic workup of breast masses. AJR 174: 1779-1783, 2000

7. Alonso O, Massado T, Delgado LB, Horvath J, Kabasakal L, Llamas-Olier A, Maunda KK, Morales R, Padhy AK, Shankar UR: Is (99m) Tc-sestamibi scintimammography complementary to conventional mammography for detecting breast cancer in patients with palpable masses? J Nucl Med 42: 1614-1621, 2001

8. Orel SG: MR imaging of the breast. Radiol Clin N Am 83: 122-126, 2000

9. Brown J, Buckley D, Coulthard A, Dixon AK, Dixion JM, Easton DF, Eeles RA, Evans DG, Gilbert FG, Graves M, Hayes C, Jenkins JP, Jones AP, Keevil SF, Leach MO, Liney GP, Moss SM, Padhani AR, Parker GJ, Pointon LJ, Ponder BA, Redpath TW, Sloane JP, Turnbull LW, Walker LG, Warren RM: Magnetic resonance imaging screening in women at genetic risk of breast cancer: imaging and analysis protocol for the UK multicentre study. UK MRI Breast Screening Study Advisory Group. Magn Reson Imaging 18: 765-776, 2000

10. Rubin HR, Gandek B, Rogers WH, Kosinski M, McHorney CA, Ware JE: Patients' ratings of outpatient visits in different practice settings. Results from the medical outcomes study. JAMA 270: 835-840, 1993

11. Olsen JA, Smith RD: Theory versus practice: a review of 'willingness-to-pay' in health and health care. Health Econ 10: 39-52, 2001

12. Frew E, Wolstenholme JL, Whynes DK: Willingness-topay for colorectal cancer screening. Eur J Cancer 37: 1746-1751, 2001

13. Wagner TH, Hu T, Duenas GV, Kaplan CP, Nguyen BH, Pasick RJ: Does willingness to pay vary by race/ethnicity? An analysis using mammography among low-income women. Health Policy 58: 275-288, 2001 
14. Blumenschein K, Johannesson M, Yokoyama KK, Freeman PR: Hypothetical versus real willingness to pay in the health care sector: results from a field experiment. J Health Econ 20: 441-457, 2001

15. Taylor SJ, Armour CL: Measurement of consumer preference for treatments used to induce labour: a willingness-to-pay approach. Health Expect 3: 203-216, 2001

16. Loeken K, Steine S, Sandvik L, Laerum E, Finset A: A new measure of patient satisfaction with mammography. Validation by factor analytic technique. Fam Pract 13: 67-74, 1996
17. Keefe FJ, Hauck ER, Egert J, Rimer B, Kornguth P: Mammography pain and discomfort: a cognitive-behavioral perspective. Pain 56: 247-260, 1994

Address for offprints and correspondence: Wenchi Liang, $\mathrm{PhD}$, Department of Oncology, Lombardi Cancer Center, Georgetown University, 2233 Wisconsin Ave, NW, Suite 440, Washington, DC 20007, USA; Tel.: +1-202-687-8937; Fax: +1-202-687-0305; E-mail: liangw1@georgetown.edu 